\title{
JMD ab 2010 bei Springer
}

\author{
Rolf Biehler • Andrea Peter-Koop • \\ Rudolf Sträßer · Hans-Georg Weigand
}

Online publiziert: 9 . Februar 2010

(C) GDM 2010

Liebe Leserin, lieber Leser des Journal für Mathematik-Didaktik,

mit diesem Heft halten Sie das erste Ergebnis nach dem Verlagswechsel der Zeitschrift Journal für Mathematik-Didaktik (JMD) in Händen. Mit dem JMD im neuen Gewand, publiziert ab 2010 im Springer-Verlag, sollen bewährte Traditionen fortgesetzt werden, aber auch neue Vorstellungen verwirklicht werden. Das JMD wird auch weiterhin die Zeitschrift der Gesellschaft für Didaktik der Mathematik (GDM) bleiben.

In Zukunft werden alle JMD-Artikel online bei Springer-Link verfügbar sein, zurückliegende Jahrgänge werden schrittweise retrodigitalisiert. Neben der PrintAusgabe erhalten alle GDM-Mitglieder einen kostenlosen Zugang zur OnlineVersion des JMD. Wir sind der Auffassung, dass wir hiermit einen wesentlichen Schritt in Richtung einer zeitgemäßen Publikationsform gehen. Die im JMD publizierten wissenschaftlichen Ergebnisse werden dadurch einfacher und schneller einem deutschen und internationalen Leserkreis zugänglich.

Das vorliegende Heft mit englischsprachigen Beiträgen und mit einem Themenschwerpunkt war schon lange vor diesem Verlagswechsel geplant. Es ist Ausdruck des Bemühens der Herausgeber des JMD und der GDM, in Deutschland gewonnene Forschungsergebnisse auch international besser darzustellen.

Dieses Heft stellt in doppelter Hinsicht einen Sonderfall dar. Zum einen werden Themenhefte beim JMD auch weiterhin die Ausnahme bleiben. Üblicherweise wird das JMD Texte zu verschiedenen Themen in einem Heft zusammenstellen. Zum anderen ist auch die Herausgabe eines Heftes in englischer Sprache ein Sonderfall. Die Herausgeber und die Gesellschaft für Didaktik der Mathematik werden auch

R. Biehler ( $\bowtie)$

Institut für Mathematik, Universität Paderborn, Warburger Str. 100, 33098 Paderborn, Deutschland e-mail: biehler@math.uni-paderborn.de 
weiterhin schwerpunktmäßig deutschsprachige Beiträge publizieren, da wir davon ausgehen, dass die deutschsprachige Didaktik der Mathematik ein Publikationsorgan braucht, in dem Mathematikdidaktikerinnen und Mathematikdidaktiker sich in deutscher Sprache, meist ihrer Muttersprache, ausdrücken können.

Diese Einschätzung soll aber die Wirkung deutschsprachiger Mathematikdidaktik in der weltweiten mathematikdidaktischen Forschergemeinschaft nicht behindern. Deshalb streben wir eine Erhöhung des Anteils englisch- und französischsprachiger Beiträge im JMD an. Wir möchten deutschsprachige Autoren ermuntern und unterstützen, ihre Beiträge im JMD in englischer Sprache zu publizieren. Umgekehrt sind Forscherinnen und Forscher außerhalb des deutschen Sprachraums herzlich eingeladen, im JMD zu publizieren.

Eine weitere Änderung ist mit diesem ersten Heft des JMD beim Springer-Verlag nicht sofort ersichtlich: Bisher erschien das JMD drei Mal im Jahr, wobei eines der Hefte immer ein Doppelheft war. Das wird sich in der Zukunft insofern ändern, als es nur noch zwei Hefte des JMD in jedem Jahrgang/jedem Jahr geben wird. Das kostengünstigere zweimalige Erscheinen der gedruckten Ausgabe wird dadurch kompensiert, dass angenommene Artikel in Zukunft sofort unter Online First verfügbar sind. Dies bedeutet, dass angenommene Beiträge der JMD-Leserschaft umgehend online zur Verfügung stehen und damit für die Autorin bzw. den Autor als publiziert eingeordnet werden dürfen. Dies überbrückt auch die nun manchmal längere Zeitspanne zwischen Annahme und Druck eines Artikels im JMD.

Unverändert bleibt die innere Organisation des JMD. Der Beirat der GDM wählt die Herausgeber auf Zeit. Die Herausgeber entscheiden auf der Grundlage von drei Fachgutachten über die Veröffentlichung eingereichter Texte. Der Beirat der GDM wählt ferner weiterhin ein Wissenschaftliches Beratungskomitee des JMD, das die Arbeit der Herausgeber unterstützt, insbesondere durch die Anfertigung von Gutachten über eingereichte Manuskripte.

Das JMD möchte weiterhin die Breite der fachdidaktischen Forschung in der GDM und darüber hinaus repräsentieren: von der Stoffdidaktik über epistemologische Analysen zur Mathematik bis hin zu quantitativer empirischer Forschung.

Wir möchten also Sie als Mathematikdidaktikerinnen und Mathematikdidaktiker weiterhin bitten, uns die Ergebnisse Ihrer Forschungen zur Publikation anzuvertrauen. Über das JMD machen Sie Ihre Texte aus der Didaktik der Mathematik einer breiten Leserschaft - zunehmend auch international - zugänglich.

Für Beiträge in englische Sprache wird der Springer-Verlag zukünftig bei der sprachlichen Umsetzung unterstützend zur Seite stehen und ggf. eine sprachliche Nachbearbeitung anbieten. Dies ist insbesondere für diejenigen von Ihnen interessant, die ihre Forschungen auch einem englischsprachigen Fachpublikum zugänglich machen möchten, bei der sprachlichen Umsetzung jedoch gern Unterstützung nutzen würden.

Insgesamt hoffen wir, dass die beschriebene Mischung aus Bewährtem und Neuem Ihre Zustimmung finden wird und freuen uns auf den Eingang zahlreicher neuer Manuskripte.

Wir bedanken uns an dieser Stelle auch sehr herzlich bei allen Gutachterinnen und Gutachtern des JMD aus dem Jahre 2009. Erfreulicherweise wurden dem JMD im letzten Jahr 33 Manuskripte eingereicht, so dass etwa 100 Gutachten zu erstellen und zu verarbeiten waren. 
Schließlich bleibt uns noch der Dank an Werner Peschek, der mit Ablauf des Jahres 2009 turnusgemäß seine 3-jährige Herausgebertätigkeit beendet. Wir hoffen, dass wir auch in Zukunft auf seinen reichen Erfahrungsschatz bei der Publikation mathematikdidaktischer Arbeiten zurückgreifen dürfen.

Paderborn, Oldenburg, Gießen und Würzburg im Januar 2010.

Rolf Biehler · Andrea Peter-Koop · Rudolf Sträßer (Herausgeber des JMD) Hans-Georg Weigand (Vorsitzender der GDM) 